\title{
THE PERMANENT OF A TRANSITIVE RELATION
}

\author{
HENRY SHARP, JR.
}

Abstract. H. Minc has constructed an upper bound on the permanent of any relation on a finite set. In this paper the permanent of any transitive relation on a finite set is calculated. The work in part is based upon the interpretation of a reflexive, transitive relation as a finite topology. The relationship to (finite) Borel fields is discussed briefly. In an example it is shown how results here may be combined with Minc's inequality to produce an improved upper bound on the permanent of any relation.

1. Introduction. Let $S=\left\{s_{1}, s_{2}, \cdots, s_{n}\right\}$ be a given finite set. A relation $\alpha$ on $S$ is a subset of $S \times S$ and will be identified with an $n \times n(0,1)$-matrix $A=\left\{a_{i j}\right\}$ in which $a_{i j}=1$ iff $\left(s_{i}, s_{j}\right) \in \alpha$. In [1], Minc established an upper bound on the permanent of any $n \times n$ $(0,1)$-matrix $A$ with row sums $r_{1}, r_{2}, \cdots, r_{n}$,

$$
\operatorname{per}(\alpha)=\operatorname{per}(A) \leqq \prod_{i=1}^{n} \frac{r_{i}+1}{2}
$$

In [2], he improved the bound as follows

$$
\operatorname{per}(A) \leqq \prod_{i=1}^{n} \frac{r_{i}+\sqrt{ } 2}{1+\sqrt{ } 2}
$$

We shall show that the bound (1) can be replaced by an exact computation in the event that $\alpha$ is transitive. It was shown in [4] that a reflexive, transitive relation corresponds to a topology on $S$. Our main results may be summarized:

per $(\alpha)=0$ if $\alpha$ is transitive but not reflexive,

per $(\alpha)=1$ if $\alpha$ corresponds to a $T_{0}$ topology,

per $(\alpha)=K>1$ if $\alpha$ corresponds to a non- $T_{0}$ topology. The evaluation of $K$ appears in Theorem 3 .

In the final section we consider, briefly, arbitrary relations $\alpha$. For relations with several maximal row and column sums, (1) may not be particularly close. Using the results above, we describe an algorithm leading to a possible improvement in the bound given by (1).

Received by the editors October 17, 1969.

AMS 1969 subject classifications. Primary 0420, 0505, 0525; Secondary 2810, 5420.

Key words and phrases. Permanent of $(0,1)$-matrix, relations on finite sets, transitive relations, finite topologies, finite Borel fields. 
2. Nonreflexive, transitive relations. Any reflexive relation necessarily has permanent $\geqq 1$. A nonreflexive relation $\alpha$ (some pair $\left.\left(s_{j}, s_{j}\right) \notin \alpha\right)$, however, may have permanent 0 . This is the case in the following transitive relation

$$
\left[\begin{array}{lll}
0 & 1 & 0 \\
0 & 1 & 0 \\
1 & 1 & 1
\end{array}\right],
$$

which provides a clue to Theorem 1 .

THEOREM 1. If $\alpha$ is a nonreflexive, transitive relation on $S$, then per $(\alpha)=0$.

Proof. Let $A=\left(a_{i j}\right)$ be the matrix corresponding to $\alpha$. By hypothesis there is an index $v$ such that $a_{v v}=0$. Now assume that $\operatorname{per}(\alpha)$ $\geqq 1$; there is then at least one permutation on the subscripts such that

$$
a_{1 i_{1}} a_{2 i_{2}} \cdots a_{n i_{n}}=1 \text {. }
$$

Factor the permutation into a product of disjoint cycles, one of which is of the form $\left(i_{k}, \cdots, i_{m}\right)$ where $i_{k}=v$. Because of $(2)$, the transitivity of $\alpha$ implies that $a_{p q}=1$ whenever $p$ and $q$ are in the same cycle, in particular when $p=q$. Thus $a_{v v}=1$, contradicting the hypothesis.

3. Reflexive, transitive relations. A reflexive, transitive relation on $S$ corresponds uniquely to a topology on $S$. To simplify notation we shall represent each by the corresponding $(0,1)$-matrix $T=\left(t_{i j}\right)$, $i, j=1,2, \cdots, n$.

Definition 1. Let $T=\left(t_{i j}\right)$ and $T^{*}=\left(t_{i j}^{*}\right)$ be topologies on $S$. The operation $\odot$ is defined by

$$
T \odot T^{*}=Y=\left(y_{i j}\right), \quad i, j=1,2, \cdots, n,
$$

where $y_{i j}=t_{i j} t_{i j}^{*}$ (Boolean product). The matrix $T \odot T^{*}$ is called the core of $T$ and $T^{*}$.

Lemma 1. The core of two topologies on $S$ is again a topology on $S$.

Proof. If $T \odot T^{*}=Y$, we need to show that $Y^{2}=Y$ (Boolean arithmetic) [4]. The $(i, j)$ th entry in $Y^{2}$ is $z_{i j}=\sum y_{i k} y_{k j}=\sum t_{i k} t_{i k}^{*} t_{k j} t_{k j}^{*}$. If $z_{i j}=1$, then for some $k t_{i k}=t_{i k}^{*}=t_{k j}=t_{k j}^{*}=1$. Hence by transitivity $t_{i j}=t_{i j}^{*}=y_{i j}=1$. If $z_{i j}=0$, then for each $k t_{i k} t_{i k}^{*} t_{k j} t_{k j}^{*}=0$. In particular, for $k=i t_{i i}=t_{i i}^{*}=1$, hence $t_{i j} t_{i j}^{*}=y_{i j}=0$.

If $B$ and $B^{*}$ are the minimal bases for the topologies $T$ and $T^{*}$, 
respectively, [4] then the nonempty members of the minimal basis for the core topology $T \odot T^{*}$ are the sets $B_{j} \cap B_{j}^{*}$.

Let $T$ and $T^{\prime}$ denote transpose topologies [4]. We shall indicate the core of $T$ and $T^{\prime}$ by $T \odot T^{\prime}=T^{c}=\left(t_{i j}^{c}\right)$, and we call it the symmetric core of $T$. It is clear that $T^{c}$ is a symmetric topology and is the matrix for an equivalence relation on $S$. Further, $T^{c}$ is the identity matrix $I$ iff the topology $T$ is $T_{0}$ [4].

THEOREM 2. If $T^{c}$ is the symmetric core of $T$, then $\operatorname{per}(T)=\operatorname{per}\left(T^{c}\right)$.

Proof. Let $E_{1}, E_{2}, \cdots, E_{m}$ be the equivalence classes into which $T^{c}$ partitions $S$. Consider an arbitrary term

$$
t_{1 i_{1}} t_{2 i_{2}} \cdots t_{n i_{n}}
$$

in the expansion defining the permanent of $T$. For each subscript pair $\left(j, i_{j}\right)$

if $s_{j}$ and $s_{i_{j}}$ are in the same equivalence class, then $t_{j i_{j}}=1$.

if $s_{j}$ and $s_{i_{j}}$ are in different equivalence classes, then $t_{j_{i j}} t_{i_{j} j}=0$.

Now factor (3) into disjoint cycles, a typical factor being of the form

$$
t_{k i_{k}} \cdots t_{m i_{m}},
$$

where $i_{m}=k$. By the transitivity of $T$, the product indicated in (4) is 1 iff $t_{k i_{k}}=t_{i_{k k}}=1, \cdots, t_{m i_{m}}=t_{i_{m} m}=1$. If each factor (cycle) involves only subscripts corresponding to elements in a single equivalence class, then $t_{1 i_{1}} t_{2 i_{2}} \cdots t_{n i_{n}}=t_{1 i_{1}}^{c} c_{2 i_{2}}^{c} \cdots t_{n i_{n}}^{c}=1$. On the other hand, if some factor (denoted as in (4)) involves a subscript pair corresponding to elements in different equivalence classes, then $t_{k i_{k}} \cdots t_{m i_{m}}$ $=t_{\boldsymbol{k}_{i_{k}}}^{c} \cdots t_{m_{i_{m}}}^{c}=0$. Thus for each term in the expansion defining the permanent $t_{1 i_{1}} t_{2 i_{2}} \cdots t_{n i_{n}}=1$ iff $t_{1 i_{1}}^{c} t_{2 i_{2}}^{c} \cdots t_{n i_{n}}^{c}=1$.

THEOREM 3. If $T^{c}$ is the symmetric core of $T$ and if $E_{1}, E_{2}, \cdots, E_{m}$ are the equivalence classes into which $T^{c}$ partitions $S$, then

$$
\operatorname{per}(T)=\prod_{k=1}^{m}\left|E_{k}\right| !
$$

Proof. The symbol $\left|E_{k}\right|$ denotes the cardinal of $E_{k}$. By Theorem 2 we need to calculate $\operatorname{per}\left(T^{c}\right)$. The relation described by $T^{c}$ is simply $\bigcup_{k=1}^{m} E_{k} \times E_{k}$. Thus

$$
\operatorname{per}\left(T^{c}\right)=\prod_{k=1}^{m} \operatorname{per}\left(E_{k} \times E_{k}\right)=\prod_{k=1}^{m}\left|E_{k}\right| !
$$


The following corollary is an immediate consequence of the remark preceding the statement of Theorem 2 .

Corollary. If $T$ represents a $T_{0}$ topology, then $\operatorname{per}(T)=\operatorname{per}(I)=1$.

4. Borel fields. The previous sections show that the permanent of any transitive relation depends upon the largest equivalence relation contained in it, being 0 unless that equivalence relation corresponds to a symmetric core topology on $S$. The symmetric core of a topology $T$ has an interpretation of interest in another context: $T^{c}$ is the Borel field generated by the topology $T[3]$. The results in $\$ 2$ of [3] are closely related to those in [4]. For example, the determinant of $T$ is 1 if the topology is $T_{0}$ and is 0 otherwise. The determinant associated with any Borel field is known, and the results here show that the permanent associated with any Borel field on $S$ is also known.

5. Arbitrary relations. It is well known that the relation $\beta$ is transitive iff $\beta^{2} \leqq \beta$ and the reflexive relation $\beta$ is transitive iff $\beta^{2}=\beta$. Now let $\alpha$ be any relation on $S$ with corresponding matrix $A=\left(a_{i j}\right)$. It is contained in a unique minimal transitive relation $\beta$, which may be found by the following iterative procedure. Define

$$
A_{0}=A, \quad A_{n}=A_{n-1}+A_{n-1}^{2}, \quad n>0 \quad \text { (again Boolean arithmetic). }
$$

If $k$ is the least integer such that $A_{k}^{2} \leqq A_{k}$, then $B=A_{k}$ is the matrix corresponding to $\beta$. Since $A \leqq B$, $\operatorname{per}(A) \leqq \operatorname{per}(B)$, hence we have the following corollary to Theorem 1 .

COROLlaRy. If the minimal transitive relation containing $\alpha$ is nonreflexive, then $\operatorname{per}(\alpha)=0$.

THEOREM 4. If the minimal transitive relation $\beta$ containing $\alpha$ is reflexive, then (in matrix notation) $\operatorname{per}(A)=\operatorname{per}\left(A \odot B \odot B^{\prime}\right)$.

Proof. By Theorem 2, per $(B)=\operatorname{per}\left(B \odot B^{\prime}\right)$, and we recall from that proof that this equality holds because a term in the expansion of $\operatorname{per}(B)$ is 0 or 1 iff the corresponding term in the expansion of $\operatorname{per}\left(B \odot B^{\prime}\right)$ is the same. Since $A=A \odot B$,

$$
\operatorname{per}(A)=\operatorname{per}(A \odot B)=\operatorname{per}\left(A \odot B \odot B^{\prime}\right) .
$$

Essentially, this theorem simply provides an algorithm which may be useful in deleting a part of $A$ which does not contribute to the permanent.

Ex́XMPLE. Applying (1) directly to 


$$
A=\left[\begin{array}{llllll}
1 & 0 & 0 & 0 & 0 & 0 \\
1 & 1 & 1 & 1 & 0 & 1 \\
1 & 0 & 0 & 1 & 0 & 1 \\
1 & 0 & 1 & 0 & 0 & 1 \\
1 & 1 & 1 & 1 & 1 & 1 \\
1 & 0 & 0 & 0 & 0 & 1
\end{array}\right]
$$

yields $\operatorname{per}(A) \leqq 38$. But in this case,

$B=\left[\begin{array}{llllll}1 & 0 & 0 & 0 & 0 & 0 \\ 1 & 1 & 1 & 1 & 0 & 1 \\ 1 & 0 & 1 & 1 & 0 & 1 \\ 1 & 0 & 1 & 1 & 0 & 1 \\ 1 & 1 & 1 & 1 & 1 & 1 \\ 1 & 0 & 0 & 0 & 0 & 1\end{array}\right]$ and $A \odot B \odot B^{\prime}=\left[\begin{array}{llllll}1 & 0 & 0 & 0 & 0 & 0 \\ 0 & 1 & 0 & 0 & 0 & 0 \\ 0 & 0 & 0 & 1 & 0 & 0 \\ 0 & 0 & 1 & 0 & 0 & 0 \\ 0 & 0 & 0 & 0 & 1 & 0 \\ 0 & 0 & 0 & 0 & 0 & 1\end{array}\right]$,

hence by Theorem $4,(1)$ yields $\operatorname{per}(A) \leqq 1$. In fact, $\operatorname{per}(A)=1$.

\section{REFERENCES}

1. Henryk Minc. Upper bounds for permanents of $(0,1)$-matrices, Bull. Amer. Math. Soc. 69 (1963), 789-791. MR 27 \#5777.

2. - An inequality for permanents of $(0,1)$-matrices, J. Combinatorial Theory 2 (1967), 321-326. MR 35 \#2765.

3. Marlon Rayburn, On the Borel fields of a finite set, Proc. Amer. Math. Soc. 19 (1968), 885-889. MR 37 \#2619.

4. Henry Sharp, Jr., Quasi-orderings and topologies on finite sets, Proc. Amer. Math. Soc. 17 (1966), 1344-1349. MR 36 \#860.

Emory University, Atlanta, Georgia 30322 